\title{
La instancia antepredicativa en la nouvelle phénoménologie
}

Jorge Luis Roggero ${ }^{2}$

Consejo Nacional de Investigaciones Científicas y Técnicas, Argentina

Recibido el 04/07/2020. Aceptado el 28/11/2020.

\begin{abstract}
Resumen
La llamada nouvelle phénoménologie ¿practica efectivamente una fenomenología? Este artículo se propone demostrar, a partir de un análisis de las propuestas de sus dos autores más representativos: Jean-Luc Marion y Claude Romano, que las obras de estos filósofos constituyen una auténtica fenomenología si se tiene en cuenta el particular estatuto que le otorgan a la instancia antepredicativa.
\end{abstract}

Palabras clave: instancia antepredicativa, lenguaje predicativo, experiencia, nueva fenomenología francesa.

\section{The Antepredicative Instance in the nouvelle phénoménologie}

\begin{abstract}
Does the so-called nouvelle phénoménologie actually practice a phenomenology? This article aims to demonstrate, based on an analysis of the proposals of its two most representative authors: Jean-Luc Marion and Claude Romano, that the works of these philosophers constitute an authentic phenomenology if the particular status they grant to the antepredicative instance is taken into account.
\end{abstract}

Keywords: antepredicative instance, predicative language, experience, new French phenomenology.

En 2002, Jean-Luc Marion sostenía que nos encontrábamos en un "momento francés de la fenomenología". Su diagnóstico no solo no ha perdido vigencia, sino que ha cobrado mayor vigor a medida que pasan los años. Ciertamente, en ningún otro ámbito se publican tantos textos ni se desarrolla tanta actividad académica relacionada con 
la fenomenología como en el ámbito francófono. Y, sin embargo, aún encontramos comentarios - en la estela de los textos seminales de Dominique Janicaud (1991 y 1998) y Jocelyn Benoist (1994, 1995 y 1998) - que cuestionan la validez fenomenológica de estos planteos. ${ }^{2}$ ¿Es posible encontrar una característica definitiva que permita inscribir las diversas propuestas de la llamada nouvelle phénoménologie en la corriente fenomenológica?

No faltan análisis que pretenden haber dado con ese rasgo. El primero de ellos es el del propio Marion en "Un moment français de la phénoménologie". El texto busca responder las críticas de Janicaud y Benoist. Marion entiende que la nouvelle phénoménologie -heredera de Lévinas, Ricœur, Henry y Derrida- es una auténtica fenomenología en tanto continúa practicando, con ciertos desplazamientos, las tres operaciones fenomenológicas principales: la reducción, la mención intencional y la constitución. El método fenomenológico sigue siendo aplicado en su rigor, pero es utilizado para aventurarse en nuevos terrenos (Cfr. Marion, 2002: 11). Es más, Marion considera que es posible identificar un horizonte común en este nuevo escenario fenomenológico, aunque considera que la idea de Janicaud de un "giro teológico" es insuficiente para denominar el "nuevo espacio de lo visible" que excede el horizonte de la objetidad (objectité) y la enticidad (étantité) (13).

En 2004, Carla Canullo publica La fenomenologia rovesciata. Allí, la autora propone una lectura de la fenomenología francesa en el primer capítulo de la obra. Los nouveaux phénoménologues comparten, además de cierta "vocación científica", una vocación de apertura a la cosa. En esta fenomenología se pone en práctica "una inversión [rovesciamento] que, lejos de invertir algunos de los temas husserlianos, se presenta como 'apertura' a las cosas mismas operada por la presión ejercida por los fenómenos" (Canullo, 2004: 23). En este sentido, si bien la idea de una fenomenologia rovesciata se propone como categoría aplicable solo a la "familia teológica" (Marion, Henry, Chrétien), también es posible entender la inversión en un sentido amplio como aplicable a la "familia merleupontiana" y a todos los nouveaux phénoménologues en general (Canullo nombra a algunos al comienzo del capítulo: Marc Richir, Henri Maldiney, Didier Franck, Jean-Luc Marion, Jean-Louis Chrétien, Michel Henry, Dominique Janicaud, Françoise Dastur, Jean-François Courtine, Jean Greisch, Philippe Capelle) (Cfr. Canullo, 2004: 22). La filósofa italiana destaca que el aporte fundamental de la fenomenologia rovesciata es señalar el carácter acontecial de lo dado (Cfr. 352).

Siguiendo los lineamientos del análisis marioniano y apoyándose en buena medida en el libro de Canullo, en 2011, Hans Dieter Gondek y László Tengelyi publican su voluminoso estudio Neue Phänomenologie in Frankreich. Según los autores, es posible encontrar un rasgo común en la vasta diversidad de obras de la generación actual de fenomenólogos franceses, y tiene que ver, como ya había indicado

2 Tal es el caso de David-François Sebbah, Emmanuel Falque, Bruce Bégout e Isabelle Thomas-Fogiel, entre otros. Sebbah afirma que la nueva tendencia de la fenomenología la hace caer "víctima de un arrebato", pues su preocupación por lo originario, su "escalada hacia lo originario", la lleva a dirigirse hacia lo que excede el campo del aparecer (Sebbah, 2001: 3). Por su parte, Falque entiende que la nueva fenomenología francesa puede caracterizarse como presa de una modalidad de "ortodoxia" que acepta y desea siempre una nueva ampliación de la fenomenicidad desde la "preferencia de lo infinito por sobre lo finito" (cfr. Falque, 2014: 16): "La preferencia cartesiana del infinito por sobre lo finito, como un derecho de prioridad ganado hace tiempo, en efecto, parece retomada hoy por buena parte de la fenomenología (de Emmanuel Lévinas a Jean-Luc Marion o Michel Henry)" (Falque, 2004: 193). Asimismo, Bégout señala que si bien los filósofos post-husserlianos logran separar con éxito a la fenomenología de la filosofía trascendental es para anexarle "otro cuerpo extraño: ontología, antropología, hermenéutica, filosofía de la vida, de la donación, del Otro" (Bégout, 2008: 132). De este modo, según Bégout, la fenomenología no puede gozar de su recién adquirida libertad, pues queda inmediatamente asociada a nueva doctrina extra-fenomenólogica. Así también, ThomasFogiel propone un análisis crítico en Le lieu de l'universel. Impasses du réalisme dans la philosophie contemporaine. La primera parte del libro diagnostica un "giro realista de la nouvelle phénoménologie". La autora sostiene dos tesis afirmables respecto de las obras de la fenomenología francesa de los últimos treinta años: 1) En todas ellas puede encontrarse un topos común que no reside en una tendencia teológica como propone Janicaud, sino en una suerte de "realismo epistemológico" fuerte. 2). Este realismo opera la disolución de la fenomenología misma (Cfr. Thomas-Fogiel, 2015: 29). 
Marion, con la superación de los horizontes de la objetidad y la enticidad. Gondek y Tengelyi entienden que, con la nouvelle phénoménologie, "ya no solo se aprehenden y se discuten fenomenológicamente los fenómenos paradójicos, hiperfenómenos o no-fenómenos, sino que se aspira a una transformación [Umwandlung] del concepto mismo de fenómeno" (2011:25). Esta transformación del concepto de fenómeno es el rasgo común: se trata de un completo cambio de paradigma dado por una nueva concepción del fenómeno como "acontecimiento de sentido" (Sinnereignis) (39).

Gondek y Tengelyi se detienen particularmente en la obra de Richir y de Marion como los dos extremos del arco de la nouvelle phénoménologie que incluye la obra de Didier Franck, Françoise Dastur, Élaine Escoubas, Jean-Louis Chrétien, Renaud Barbaras, Natalie Depraz y Jocelyn Benoist. En todos estos fenomenólogos puede constatarse el intento de dar cuenta de cierto "exceso no objetivo" (ungegenständlicher Überschuss) presente en el corazón del fenómeno. Situándose más allá del horizonte de la fenomenología husserliana y heideggeriana, todos estos autores sostienen la afirmación del "carácter acontecial" (Ereignisscharakter) de lo dado (670-672).

Si bien hay diferencias entre estos diagnósticos y algunos aspectos pueden ser cuestionables, considero que todos ellos señalan en una dirección que debe ser explorada. La absoluta iniciativa que el nuevo estatuto acontecial otorga al fenómeno exige indagar en su relación con la instancia antepredicativa. Este artículo se propone afirmar que es posible encontrar un rasgo fenomenológico decisivo presente en la nouvelle phénoménologie en el estatuto que ella le confiere a esta instancia. Claude Romano acierta al considerar que:

\section{si hay una tesis que parece compartida por casi todos los fenomenólogos, quizás incluso la única, es aquella según la cual los fenómenos se nos presentan con un sentido autóctono que no es proyectado sobre ellos por nuestros esquemas lingüísticos (Romano, 2010: 172).}

Sin duda, hacer fenomenología implica aceptar el carácter derivado de los significados lingüísticos y la primacía de la instancia antepredicativa como portadora de un sentido propio originario, fundador de todo sentido. En ese gesto radica, como bien destaca Romano, "el primer paso de la fenomenología, el que le da su desarrollo, su impulso y su orientación fundamental" (2010: 172). No obstante, las indagaciones de Husserl en el ámbito prelingüístico no parecen tener la misma finalidad que la de los "nuevos fenomenólogos". Es necesario, entonces, advertir la especificidad del estatuto de la instancia antepredicativa en la nouvelle phénoménologie, como continuación del impulso husserliano y como el rasgo definitivo que permite hablar de una nueva fenomenología en Francia.

A tal fin, teniendo en cuenta que un examen detenido de cada uno de los autores que componen el amplio campo de la nueva fenomenología francesa excedería los límites de extensión de un artículo y -en algún sentido- se revelaría redundante, me limitaré a sugerir la viabilidad de esta hipótesis exponiendo las ideas de los dos autores vivos más representativos de este "momento": Jean-Luc Marion y Claude Romano. En un primer apartado, me detendré en la noción husserliana de lo antepredicativo. En un segundo apartado, presentaré la propuesta de Romano como continuadora de la exigencia fenomenológica husserliana. En un tercer apartado, introduciré el aporte de Marion respecto de la necesidad de una indagación en otros actos lingüísticos además del enunciado predicativo. Finalmente, extraeré algunas conclusiones. 


\section{La instancia antepredicativa en Husserl}

Como es bien sabido, Husserl propone explícitamente una investigación de la instancia antepredicativa como fundadora de la predicativa en Erfahrung und Urteil: "Una teoría de la experiencia antepredicativa, precisamente de aquella que ofrece los sustratos más originarios en una evidencia objetiva, constituye en sí el primer elemento de la teoría fenomenológica del juicio" (Husserl, 1939: 21). Esta es la tarea que emprende su "genealogía de la lógica", al abordar el problema del origen del juicio predicativo (cfr. Husserl, 1939: 1). En este sentido, Husserl aclara el alcance de su indagación en la "Introducción":

Un objeto como posible sustrato de un juicio puede estar dado con evidencia sin que tenga que ser juzgado en un juicio predicativo. Pero un juicio predicativo evidente acerca de él no es posible sin que él mismo esté dado con evidencia. A primera vista, esto no tiene nada de extraño para los juicios basados en la experiencia; es más, al señalarse esta fundamentación de la evidencia predicativa en una evidencia antepredicativa, solo parece expresarse un lugar común. Pero el retroceso a la evidencia objetiva antepredicativa adquiere su peso y todo su significado solo cuando se establece que esta relación de fundamentación no solo se refiere a los juicios basados en la experiencia, sino a todo posible juicio predicativo evidente en general y, por lo tanto, también a los juicios del lógico mismo con sus evidencias apodícticas que, según vimos, pretenden valer "por si". y sin considerar su posible aplicación a un determinado ámbito de sustratos (12-13).

Es en el ámbito antepredicativo donde debe buscarse la fundamentación, no solo para los juicios basados directamente en la experiencia, sino también para todo juicio predicativo en general.

Ahora bien, este proyecto no constituye una excepción en la obra husserliana, sino que la caracteriza como un rasgo propio desde las tempranas Logische Untersuchungen. ${ }^{\text {S }}$ Ya en la Introducción, Husserl señala: "Los conceptos lógicos como unidades válidas de pensamiento deben tener su origen en la intuición" (Hua XIX/1: 10). Pero, particularmente, en la Sexta Investigación, Husserl procura fundar la evidencia predicativa

3 Esta misma afirmación puede encontrarse en el § 86 de Formale und Transzendentale Logik: “...la teoría del juicio primera en sí es la teoría de los juicios evidentes, y lo primero en sí en una teoría de los juicios evidentes (y por ende en una teoría del juicio en general) es la remisión genética de las evidencias predicativas a la evidencia no predicativa, que se llama experiencia" (Hua XVII: 217).

4 En todos los casos se tuvieron a la vista las traducciones al español existentes y se introdujeron modificaciones cuando se consideró pertinente.

5 Como bien destaca Bruce Bégout, la investigación en la "genealogía de la lógica", propuesta en Erfahrung und Urteil, puede ser caracterizada como una auténtica "arqueología fenomenológica" (Hua Mat VIII: 356), según la expresión utilizada en los Manuscritos C. Efectivamente la intención de Husserl no se limita a indagar en el desarrollo de la lógica, sino principalmente en su origen. "En este sentido, la génesis de la lógica (la genealogía propiamente dicha) puede ser definida como la reconducción del pensamiento a su lugar de emergencia, a sus principios primeros" (Bégout, 2000:242). Bégout subraya que esta caracterización "arqueológica" del proyecto husserliano ya puede encontrarse en Erste Philosophie. Alli, Husserl define a la fenomenología en el sentido de una "filosofía primera" como una "ciencia de las fuentes originarias" (Urquellewissenschaft) (Hua VIII: 4) y como una "arqueología" (29). Husserl entiende que "es necesaria una ciencia que, como lo que verdaderamente podríamos llamar una 'arqueología', explore ese origen último y todos los orígenes de lo que decide sistemáticamente respecto del ser y la verdad en sí, y que a continuación nos enseñe cómo, a partir de esta fuente original de todas las opiniones y validez, cualquier conocimiento pueda ser llevado a la forma de la razón más alta y última, la del fundamento absoluto y la justificación absoluta, el esclarecimiento del sentido concebido de modo último y la ejecución de su derecho" (29-30). Como puede observarse, la preocupación por indagar en los fundamentos de la lógica en una instancia experiencial anterior atraviesa toda la obra de Husserl.

6 En Au cœur de la raison, la phénomenologie, Romano dedica buena parte del capítulo IV a un análisis de las Logische Untersuchungen destacando que uno de los propósitos de este texto de Husserl es dar cuenta del modo en que el lenguaje puede operar como el organon para la descripción de fenómenos y para la teoría del conocimiento (cfr. 2010: 137). Teniendo en cuenta que el lenguaje no es, para Husserl, un "conjunto de creencias justificadas", sino una "captación intuitiva y, en algunos casos, evidente de su objeto" (138), Romano señala que "para que el sentido de las expresiones se ajuste o coincida con los objetos susceptibles de ser dados a la conciencia [...] es necesario que la intuición posea su propio orden de sentido" (id.). Esta consideración guía su lectura respecto del modo en que se articula la relación entre expresión, significación y objeto en esta obra temprana de Husserl. Romano destaca que existe una tensión fundacional en la fenomenología "entre la afirmación de que el lenguaje está por completo al servicio de un conocimiento intuitivo del mundo, y la idea de que las cosas recogidas en la experiencia antepredicativa despliegan una 'lógica' más originaria que la que estructura nuestra inteligencia discursiva: una lógica que, si bien no es refractaria del lenguaje, tampoco deriva de él" (139). No obstante, más allá de algunas vacilaciones producto de esa tensión, Romano sostiene que cabe concluir, como un rasgo decisivo de la propuesta husserliana, la afirmación de que "el cumplimiento intuitivo depende de nuestra conciencia prelingüística del mundo, reside en un 'sentido' que precede por derecho a su expresión..." (171). 
en la evidencia perceptiva. El objetivo del texto es repensar el alcance del criterio de evidencia introducido en la Primera Investigación. Husserl se pregunta, en el § 40, cómo es posible el cumplimiento intuitivo de las formas categoriales del lenguaje:

En el caso del enunciado de percepción no se cumplen solamente las representaciones nominales entretejidas en él; lo que encuentra cumplimiento por medio de la percepción subyacente es la significación enunciativa en su conjunto. Del enunciado entero se dice igualmente que da expresión a nuestra percepción; no decimos meramente veo este papel, un tintero, varios libros, etc., sino también veo que este papel está escrito, que aquí hay un tintero de bronce, que varios libros están abiertos, etc. Si el cumplimiento de las significaciones nominales parece suficientemente claro a cualquiera, entonces hacemos esta pregunta: ¿Cómo debe entenderse el cumplimiento de los enunciados enteros, principalmente en lo que trasciende de su "materia", es decir, de los términos nominales en el presente caso? ¿Qué es lo que da y puede dar cumplimiento a esos momentos de la significación, que constituyen la forma de la proposición como tal, a los momentos de la "forma categorial" [kategoriale Form] —a los cuales pertenece, por ejemplo, la cópula? (Hua XIX/2: 657-658).

Husserl advierte con agudeza que aun cuando los estados de cosas categoriales no se correspondan con objetos sensibles, presentan un tipo de evidencia similar. ¿Cómo es esto posible? El fundador de la fenomenología va a responder introduciendo la noción de "intuición categorial" (kategoriale Anschauung). Mediante una ampliación de la percepción, Husserl explica la posibilidad de un cumplimiento intuitivo del enunciado, con sus expresiones categoriales, distinguiendo dos tipos de actos. En la estructura de la intencionalidad se encuentran actos fundantes (fundierende Akte) que se corresponden con la percepción sensible y actos fundados (fundierte Akte), que solo pueden darse sobre la base de los actos fundantes, y se corresponden con la percepción categorial. ${ }^{7}$ Husserl acepta la crítica kantiana al argumento ontológico: el "ser" no es un predicado real. Esto lo lleva a sostener que los correlatos intencionales de los actos fundados no son momentos reales de los objetos dados en la percepción sensible. Pero este correlato tampoco debe ser buscado, a la manera de un psicologismo, en la reflexión sobre los contenidos de la experiencia interna. Explica Husserl:

El origen de los conceptos de situación objetiva y de ser (en el sentido de la cópula) no está verdaderamente en la reflexión sobre los juicios, o más bien, sobre los cumplimientos de los juicios, sino en los cumplimientos mismos de los juicios; el fundamento de la abstracción, por medio de la cual realizamos dichos conceptos, no se encuentra en estos actos tomados como objetos, sino en los objetos de estos actos (Hua XIX/2: 669-670).

Los actos categoriales operan de tal forma que lo dado en la percepción sensible, sin ser modificado en su contenido, adquiere una nueva objetividad. Como bien señala Heidegger, esto implica que

todo lo categorial se apoya en última instancia en la intuición sensible, toda explicación objetual no es algo que flote en el aire [freischwebende], sino que lo es respecto de lo dado de antemano. Esta tesis de que todo lo categorial se apoya en última instancia en la intuición sensible no es sino otra formulación

7 Cabe aclarar que la distinción acto fundante/acto fundado es más amplia que la distinción percepción sensible/percepción categorial. Husserl contempla la posibilidad de actos fundados no categoriales, como los afectivos o los actos prácticos de la voluntad. Es más, en el §12 de Erfahrung und Urteil Husserl distingue entre experiencias simples y experiencias fundadas (Husserl, 1939: 54), es decir, aplica la distinción fundante/ fundado al propio nivel de la experiencia, sobre el cual se fundan luego otros actos, incluidos los predicativos. 


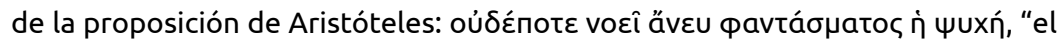
alma no puede aprehender lo objetual en su objetualidad, nada puede pensar, si no se le ha mostrado antes algo". Un pensar que no tenga fundamento en la sensibilidad es un contrasentido (GA 20: 94).

Desde sus comienzos, la fenomenología husserliana afirma la prioridad de la experiencia como instancia antepredicativa respecto de su articulación lingüística.

Ahora bien, ¿cómo cabe entender la especificidad de la relación entre el acto fundado y el acto fundante? La intuición categorial presupone un acto perceptivo, que da leibhaftig al objeto respecto del cual es posible la predicación categorial. Para que el enunciado "este papel es blanco" pueda tener evidencia categorial, es necesario que los objetos "papel" y "blanco" sean previamente dados en un acto de percepción sensible. De este modo, como bien señala Bégout, la intuición categorial es fundada porque se vale de la intuición sensible como su "fundamento condicionante" (Bégout, 2000: 263). Pero ¿cuál es el alcance de esta relación de fundamentación? Discutiendo la tesis de Dieter Lohmar en "La genèse du jugement antéprédicatif dans les Recherches Logiques et dans Expérience et Jugement", Bégout señala que la relación entre ambos actos es meramente formal. En primer lugar, esto es así porque el acto fundado no cumple al acto fundante, sino que solo funciona como su condición de cumplimiento. Pero, además, en segundo lugar, esto es así porque, a diferencia de las conexiones sensibles que son "momentos de los objetos reales" (Hua XIX/2: 684), las conexiones categoriales solo existen en el modo en que se aprehende el objeto (cfr. Bégout, 2000: 266-267). 'Y es precisamente este carácter formal de la fundamentación la que da lugar a una revisión en Erfahrung und Urteil. Bégout acierta al señalar que la relación entre lo sensible y lo categorial en las Logische Untersuchungen se limita a la de la estructura lógica de presuposición entre antecedente y consecuente porque todavía no fue introducida la perspectiva genética (cfr. 270).

Con Erfahrung und Urteil, la relación de fundamentación se transforma porque, desde un punto de vista genético, los sustratos individuales de la instancia antepredicativa comienzan a operar como elementos pre-constitutivos en un sentido material y no meramente formal. Husserl señala que la evidencia última puede encontrarse en la evidencia experiencial, en la evidencia de estos sustratos individuales. ${ }^{11}$ Ahora bien, aun respecto de estos sustratos originarios cabe preguntarse si éstos son dados de modo apodíctico y completamente adecuado. Husserl responde que no, pues en tanto se trata de objetos dados a la percepción externa se inscriben en un horizonte

\footnotetext{
8 Lohmar considera que la síntesis perceptiva de actos parciales en la unidad perceptiva opera no solamente como "fundamento" para el acto categorial, sino que también funciona como "el contenido que cumple la intención categorial" (Lohmar, 1996: 222). Bégout destaca que esto implica ir más allá de la "férrea distinción que Husserl había introducido entre lo sensible y lo categorial" (Bégout, 2000: 268). Cabe preguntarse cuál es la posición de la nouvelle phénoménologie respecto de esta "férrea distinción" y de la ampliación de la intuición llevada a cabo por Husserl. En el primer capítulo de Réduction et donation, Marion reflexiona sobre las lecturas de las Logische Untersuchungen de Husserl y de Derrida para concluir: "Lo que está en juego en las Investigaciones, particularmente en Vla, se debe menos a la intuición categorial que a lo que ella indica, sin realizarla ella misma: la ampliación de la presencia entendida como objetividad, según la medida excesiva de la donación. La ampliación debe entenderse no solo como una extensión (Erweiterung) de la intuición y de la significación por la donación, sino sobre todo como 'la exigencia de una liberación del suelo (Freilegung des Bodens)' fenomenológico. [...] Parece lícito suponer que Husserl, como sumergido por el imperativo, amenazante y jubiloso al mismo tiempo, de administrar la sobreabundancia de lo dado en presencia, no se interrogue en ningún momento (al menos en las Investigaciones lógicas) acerca del estatuto, el alcance y aun la identidad de la donación" (1989: 60 y 62). Según Marion, la analogía que Husserl establece entre la intuición categorial y la intuición sensible le impide advertir la absoluta liberación de la donación que esta ampliación implica.

9 En este sentido, Romano destaca que, a diferencia de Reinach, Husserl nunca sostuvo que los "estados de cosas" tengan una existencia independiente del juicio que los identifica. Un estado de cosas constituye un tipo de objeto que solo puede darse en relación con un ente dotado de lenguaje (cfr. 2010: 164).

10 Cabe destacar que no hacemos referencia a los análisis de los §§ 124-127 de Ideen I porque allí podemos encontrar el mismo tipo de relación formal que caracteriza a las reflexiones de la fenomenología estática de las Logische Untersuchungen. En esos parágrafos se encuentra una conocida afirmación que resume de modo elocuente la subordinación del lenguaje a la experiencia: “La capa de la expresión no es productiva [Die Schicht des Ausdruckes ist (...) nicht produktiv]" (Hua III/1: 287).

11 “...los juicios primarios en si como juicios con sustratos individuales, los juicios acerca de lo individual, son los juicios de la experiencia. La donación [Gegebenheit] evidente de objetos individuales de la experiencia, es decir, su donación antepredicativa, precede a esos juicios. Por consiguiente, la evidencia experiencial sería la evidencia originaria última buscada por nosotros y, de este modo, el punto de partida para el esclarecimiento del origen del juicio predicativo" (Husserl, 1939: 21).
} 
de donación infinito: "toda cosa experimentada tiene no solo un horizonte interno, sino también un horizonte externo, abiertamente ilimitado, de objetos que se dan junto a él" (Husserl, 1939: 28). De este modo, la certeza de estos sustratos últimos es puesta en cuestión toda vez que este horizonte es abierto y que conjuga lo conocido con lo desconocido. ${ }^{12}$ Por este motivo, Husserl va a desplazar la certeza última a la pre-donación del mundo.

Este suelo universal de la creencia en el mundo está presupuesto por toda praxis, tanto por la praxis de la vida como por la praxis teórica del conocimiento. El ser del mundo en su totalidad es lo sobreentendido, de lo que nunca se duda y que no ha sido adquirido primero mediante la actividad judicativa, sino que ya es presupuesto para todo juicio (25).

Este suelo originario, que constituye el "mundo de la vida", va a estar dotado de una lógica propia. Husserl sostiene en la Krisis que este estrato antepredicativo tiene una cientificidad propia, "una que precisamente no es lógica-objetiva pero que como fundante en sentido último, respecto del valor, no resulta menor sino superior" (Hua VI: 127$).^{13}$ La génesis de la lógica predicativa yace en esta lógica antepredicativa, esta lógica sensible que la fenomenología debe desentrañar y de la que extrae todo sentido.

\section{La tesis fenomenológica según Romano}

La obra de Claude Romano enfatiza particularmente este aspecto de la fenomenología husserliana. En Au couur de la raison, la phénoménologie (2010), Romano destaca la especificidad de la noción fenomenológica de "sentido". A diferencia de la filosofía analítica, que entiende que la significación y el sentido se circunscriben a la esfera lingüística, la fenomenología refiere a un "sentido inherente a la experiencia" (Romano, 2010: $135){ }^{14} \mathrm{Y}$ es hacia este sentido al que dirige todo su esfuerzo: se trata de aprehenderlo en sus propios términos. Romano sostiene que Husserl define el objetivo de todo su proyecto filosófico en una frase de las Cartesianische Meditationen: "el comienzo es la experiencia pura y, por así decirlo, todavía muda, a la que ahora hay que llevar a la expresión pura de su propio sentido [ihres eigenen Sinnes]" (Hua I: 77). Se trata, pues, de encontrar la Sprachlichkeit adecuada al sentido que ya se encuentra en la experiencia. Este es precisamente, según Romano, el contenido de la exigencia más propia de la fenomenología.

El voluminoso libro de Romano presenta su ambicioso proyecto desde el comienzo: su propósito es ofrecer una introducción a la fenomenología que, a su vez, oficie de justificación de sus tesis principales (Romano, 2010: 18). En el Prefacio, Romano destaca la importancia de advertir el "problema fenomenológico". Este radica en determinar si efectivamente la experiencia posee estructuras inmanentes necesarias y autónomas respecto de las reglas instituidas del lenguaje y los esquemas conceptuales (cfr. 2010: 11). No puede aceptarse sin más las implicancias del "giro lingüístico". La indagación filosófica en la experiencia no es una tarea que haya perdido su vigencia. En este sentido, según el autor, es necesario sostener la siguiente "tesis fenomenológica":

12 "Posee, pues, su horizonte vacío de un desconocimiento conocido" (Husserl, 1939: 35).

13 Husserl distingue expresamente entre un a priori lógico-objetivo y un a priori del mundo de la vida (cfr. Hua Vl: 140-145).

14 En las elocuentes palabras de Romano: “Tome el caso de un filósofo, examine el empleo que hace del término 'sentido' e inmediatamente usted no tendrá dudas: si solo habla de dar un sentido a un signo y de explicar el sentido de una frase, usted tiene un filósofo analítico; si emplea 'sentido' de una manera más amplia usted está tratando con un discípulo de Husserl" (Romano, 2010: 135-136). 
hay una autonomía del orden prelingüístico de la experiencia "antepredicativa", como habría dicho Husserl, respecto de las formas superiores de pensamiento y el lenguaje. La experiencia posee un lógos inmanente que la fenomenología, precisamente, tiene por propósito sacar a la luz. Es más, el lenguaje mismo solo deviene plenamente inteligible si se lo restituye a su relación con una inteligencia prelingüística (2010: 12).

Por este motivo, Romano afirma a continuación que es indispensable poner en cuestión cierto "marco kantiano" imperante en la filosofía contemporánea. Este "marco" consiste en "concebir todo orden y toda estructuración de la experiencia como extrínseca a ella, como surgida del lenguaje, de la cultura o de sus esquemas" (2010: 13). La originalidad de la noción husserliana de experiencia consiste en situarse más allá del debate entre el empirismo y el kantismo. Según el fenomenólogo francés, el "marco kantiano" de la filosofía contemporánea puede observarse en cierta estrategia que sitúa al lenguaje en el lugar de las categorías kantianas: gran parte de la filosofía analítica, como el neokantismo criticado por Husserl, opera con este tipo de "construcciones trascendentales desde arriba [transzendentale Konstruktionen von oben]" (Husserl, 2013: 115) que olvida que "la experiencia, con sus exigencias propias precede al pensamiento conceptual y sus exigencias" (Hua V: 34).

Ahora bien, para sostener la tesis fenomenológica frente a la proliferación de propuestas filosóficas que sostienen el "marco kantiano" es preciso detenerse en el estatuto de la experiencia y del lenguaje, y esto, explica Romano, implica una indagación en la razón misma. Por este motivo, la fenomenología propone una "nueva imagen de la razón". Husserl afirma un lógos inmanente a lo sensible, un "lógos del mundo estético" (Logos der ästhetischen Welt) (Hua XVII: 297). En consecuencia, ya no cabe asociar la experiencia con la irracionalidad. Hay que evitar caer en una "racionalidad de corazón estrecho" (enghertzige Rationalität) (Hua VI: 14). Existe una racionalidad propia de la experiencia. En este sentido, Romano enfatiza que ser fiel al proyecto fenomenológico exige afirmar "una razón ampliada hasta incluir a su otro: la sensibilidad, la experiencia, lo antepredicativo, una razón de gran corazón (raison au grand cœur) que rehabilite el mundo sensible como necesario para su propia existencia" (Romano, 2010: 14-15), es decir, una racionalidad capaz de captar la lógica del sentido inherente a la experiencia. Esta es la tarea de la fenomenología.

Cabe destacar que puede encontrarse este acento puesto en la "tesis fenomenológica" desde los comienzos de la obra de Romano. En la tercera parte de L'événement et le monde, destinada a indagar en la noción fenomenológica de "experiencia", Romano distingue dos modos de concebirla relacionados con dos verbos: avoir (tener) y faire (hacer). El empirismo tiene una concepción de la experiencia relaciona con el verbo avoir. En este sentido, la experiencia parece ser algo que puede ser acumulado y constituir un acervo de conocimiento adquirido, una posesión. La fenomenología, según Romano, sostiene un concepto de experiencia relacionado con el verbo faire.

La experiencia que hago constituye una prueba única, irrepetible, en la que me pongo en juego a mí mismo y de la que resurjo, cada vez, cambiado; lo que prima aquí no es la idea de adquisición, sino al contrario la de una puesta a prueba que

15 Cabe señalar que esta tesis ya era sostenida en el ámbito de la fenomenología francesa por Paul Ricœur. En su célebre "Phénoménologie et herméneutique. En venant de Husserl", el hermeneuta francés destaca como fundamental para la fenomenología (y para su articulación con la hermenéutica) la afirmación de la tesis del carácter derivado de los significados del orden lingüísticos. "Esta remisión del orden lingüístico a la estructura de la experiencia (que en el enunciado llega al lenguaje) constituye, a mi juicio, el presupuesto fenomenológico más importante de la hermenéutica" (1975: 67). 
es a la vez transformación: no puedo hacer una experiencia más que si ella, de manera insustituible, me sucede, dándome imprevisiblemente como porvenir a mí mismo, siempre nuevo y siempre otro (1998: 194-195).

Romano busca apoyo a estas ideas en un pasaje de Heidegger en Das Wesen der Sprache:

Hacer una experiencia [eine Erfahrung machen] con algo, ya se trate de una cosa, de un hombre, de un dios, quiere decir: que eso nos ocurra, nos alcance, nos sobrevenga, nos dé vuelta y nos transforme. Cuando hablamos de 'hacer' una experiencia esto no significa precisamente que nosotros la realicemos; hacer significa aquí: atravesar, sufrir, tomar lo que nos alcanza receptivamente, aceptar, en la medida en la que nos sometemos a algo (GA 12: 149).

Según Romano, la experiencia, la auténtica, la fenomenológica, es aquella que nos atraviesa más allá de nuestra capacidad de preverla y controlarla. "Hacemos" una experiencia cuando somos capaces de entregarnos al acontecimiento del advenir del fenómeno en sus propios términos, sin imponerle condiciones. Así, la experiencia, en su sentido primero, al constituir una instancia de exposición, es aquello que nos afecta, que nos transforma. ¿Y a qué nos exponemos? Al sentido del fenómeno que se da en la experiencia, antes que cualquier posible articulación lingüística.

\section{Los actos lingüísticos no-predicativos según Marion}

En un pasaje del capítulo IV de Au cœur de la raison, la phénoménologie, Romano señala lúcidamente que, al restringir la indagación al origen del lenguaje predicativo y sus enunciados asertivos, al considerar al lenguaje solo como un medio de formulación y comunicación de conocimientos, Husserl excluye de su investigación otros actos lingüísticos tales como la orden, la promesa o la plegaria (cfr. Romano, 2010: 156). En este sentido, cabe precisar qué debe entenderse como "la experiencia antepredicativa" (vorprädikative Erfahrung). El adjetivo vorprädikative: "antepredicativo" o "prepredicativo" ya limita la investigación en la experiencia a aquellos sentidos que puedan dar lugar a un lenguaje predicativo. ${ }^{16}$ En este sentido, habría que hablar de lo "pre-lingüístico", más que de lo "antepredicativo". Y este es precisamente el camino que recorre la indagación de la nouvelle phénoménologie, que 1) ya no busca reconducir los sentidos inherentes a la experiencia al plano teórico de la lógica, sino que intenta dar cuenta de la validez de ellos en el ámbito de la práctica, y que 2) ya no restringe su investigación en la instancia prelingüística al origen de los enunciados predicativos, sino que indaga en la génesis de otros actos lingüísticos. Es más, a partir de la obra levinasiana, se comienza a prestar atención a la centralidad

16 El propio Heidegger ya había advertido esta limitación en el § 73 de su curso de 1929, Die Grundbegriffe der Metaphysik: "La forma del enunciado en el sentido del enunciado positivo verdadero facilita -por motivos que ahora no tratamos- la interpretación del lógos. Este tipo de planteamiento de la lógica en el caso del juicio positivo verdadero está justificado dentro de ciertos límites, pero justamente por ello pasa a ser ocasión de la confusión fundamental, como si se tratara solo de referir simplemente las restantes formas posibles del enunciado a la mencionada, para completarla. Yo mismo -al menos en el desarrollo de la interpretación del lógos- fui víctima de esta confusión en Sery tiempo (cfr. como excluido de esta confusión Ser y tiempo, p. 222 y pp. 285ss.). En la interpretación que hay que dar ahora, que ciertamente no conculca en realidad lo expuesto antes en Ser y tiempo, me apartaré esencial y decisivamente de ello" (GA 29/30: 488-489). Para una lectura de las implicancias de esta crítica de Heidegger y el modo en que con ella se reestructura la relación entre lo antepredicativo y lo predicativo, cfr. Ainbinder y Stanciu, 2020. No obstante, cabe señalar que sobre el final del § 86 de Formale und Transzendentale Logik, Husserl indica: "En otra parte deberá desarrollarse la correspondiente exposición, de ninguna manera fácil, de la multiforme operación de experiencia que se cumple en el juicio de experiencia; exposición también de ese mismo juicio original. Enfaticemos en particular solamente que incluso esta experiencia fundante tiene sus propios modos de operaciones sintácticas; pero éstas aún están libres de todas las conformaciones conceptuales y gramaticales que caracterizan lo categorial en el sentido del juicio predicativo y del enunciado" (Hua XVII: 220). De este modo, Husserl parece dejar abierta la posibilidad de investigación respecto de otros actos lingüísticos. En una nota al pie aclara: "En mis Logische Untersuchungen, segunda parte, Vla Investigación, se introdujo por primera vez el concepto de 'categorial' referido exclusivamente a los componentes sintácticos del juicio. Todavía no se distinguía entre lo sintáctico en general que ya aparece en la esfera antepredicativa y por lo demás tiene también sus análogos en la esfera afectivo-volitiva [seine Analoga im Gemüt hat], y lo sintáctico de la específica esfera del juicio"'” (220 n. 2). 
lingüística del vocativo, del apóstrofe como fundador de las demás expresiones del lenguaje (incluidas, obviamente, las predicativas).

La manifestación del Otro en el rostro, dice Levinas, es lenguaje sin palabras, es el lenguaje originario que se da en el modo imperativo de la llamada. Según Levinas, la "relación metafísica", la relación entre lo Mismo y lo Otro es el lenguaje, pues el lenguaje constituye un dirigirse a otro en el que éste no pierde su transcendencia (cfr. Levinas, 1961: 28-29). Esta "relación metafísica" "está antes de la proposición negativa o afirmativa, instaura el lenguaje en el que ni el "no" ni el "sí" son la primera palabra" (32). La expresión lingüística fundamental no es la proposición predicativa, sino el apóstrofe. La primera palabra, la que instaura esta relación, consiste en un dirigirse a otro, tiene la forma de una llamada, de una interpelación, de un mandamiento que se manifiesta en el rostro del Otro.

La concepción levinasiana del lenguaje afirma que todo acto lingüístico tiene su origen en una experiencia, la de la relación metafísica, que, además, ya tiene en sí misma el carácter de un lenguaje no-predicativo, un lenguaje pragmático. El sentido prelingüístico fenomenológico que funda el sentido lingüístico se manifiesta en la estructura misma del lenguaje, que expresa la relación metafísica entre lo Mismo y lo Otro. Marion asume esta concepción lévinasiana al aceptar que en la indagación respecto del lógos "se trata de pasar de la predicación al dirigirse a..." (Marion, 2012: 279). Levinas sugiere: "La esencia del discurso es la oración" (Levinas, 1951: 19). La oración es la esencia del discurso porque el lenguaje, en su origen, refiere al apóstrofe de la relación ética, que se da en términos ciertamente no-predicativos, en el mero dirigirse a un Otro. En La rigueur des choses, Marion señala la particularidad de la oración:
La pragmática comenzó a describir los juegos del verbo, pero la filosofía todavía se preocupa principalmente de la predicción y del trabajo que ella siempre hace en favor de la objetidad; ella apenas sospecha que la función fundamental de lo que ya no debería llamarse lenguaje, ni siquiera lengua, sino verbo, se ejerce en la palabra, en el acto de dirigirse. El dirigirse o el verbo dirigido hacia otro, cargado de ningún objeto, que puede, por tanto, hacer lo que dice al decirlo, que coincide consigo mismo lo suficiente como para verificarse a sí mismo. [...] ¿Qué lógos permanece siendo un lógos, sin no obstante movilizarse al servicio de la predicación? Aristóteles respondió a esta pregunta en el tratado De la interpretación: un lógos que no dice nada de nada, pero que aún declara, esto se llama oración. Pues, de hecho, la oración se dirige, dirige un dirigirse a alguien; la oración o el lógos dirigido (Marion, 2012: 244-245).

Ciertamente, el interés marioniano por la oración tiene que ver con encontrar un lenguaje capaz de expresar la saturación, un lenguaje adecuado a la experiencia de fenómenos no objetivables. ${ }^{17}$ Esta búsqueda aparece esbozada en diversos pasajes de su obra. En Au lieu de soi, Jean-Luc Marion explora la alabanza como otro dispositivo lingüístico que se sustrae a la predicación y, por lo tanto, al uso teórico del lenguaje, dando lugar a un uso pragmático:

Por medio de una extraordinaria ruptura con el modo de hablar metafísico, como la predicación de algo sobre algo, la alabanza ya no pretende decir nada sobre Dios, sino que significa, precisamente, que no digo nada sobre Dios, o más 
bien, significa para Dios que lo reconozco como el único Dios, al decírselo y al reconocerme a mí mismo como no-dios (Marion, 2008: 39).

En este mismo sentido, en Certitudes négatives, analiza el modo en que el lenguaje predicativo está asociado con el conocimiento y la certeza afirmativa:

La equivalencia entre certeza y conocimiento también puede cuestionarse. Y debe hacérselo, pues puede hacérselo. Es posible encontrar también, en efecto, conocimientos sin certeza, pues carecen de objeto. La certeza trabaja, ciertamente, en primer lugar y la mayor parte del tiempo, con objetos y a la vista de objetos. Ella se alcanza ejerciendo el uso predicativo y, por tanto, afirmativo (categórico) del lenguaje: hablar consiste en hablar para decir alguna cosa (no hace falta hablar para no decir nada, nos lo han dicho bastante)... hablar para decir alguna cosa equivale a decir alguna cosa de alguna cosa, atribuir una propiedad a un sujeto: el cielo es azul, la tierra es redonda, 2 y 2 son 4 , aquél que duda es, Dios no es visible, el futuro es desconocido, etc. Incluso si todas estas afirmaciones no se verifican, incluso si no desembocan todas en la constitución de un objeto, su certeza eventual resultará de su ambición categórica. Su certeza eventual será siempre afirmativa. Tal parece ser el caso de los enunciados de las ciencias exactas, según su propia y constante pretensión: decir alguna cosa cierta sobre alguna cosa, por medio de un enunciado afirmativo (2010: 14).

Marion se pregunta si es posible algún tipo de certeza respecto de una experiencia no objetivable, una experiencia en la que es dado un sentido que se resiste a su objetivación, y responde introduciendo la noción de "certeza negativa", una certeza que se hace presente en la experiencia, pero no puede ser expresada en el lenguaje predicativo afirmativo, que exige la exploración de otro tipo de lenguaje."

Puede afirmarse, entonces, que la fenomenología marioniana constituye una auténtica fenomenología en tanto continúa siendo fiel al mandato husserliano más decisivo, que consiste en afirmar la "tesis fenomenológica". Pero, además, puede sostenerse que continuando los lineamientos del fundador de la fenomenología, al descubrir la complejidad de la instancia prelingüística, que no siempre se deja reconducir a la objetidad y al lenguaje predicativo, Marion se preocupa por indagar en diversos actos lingüísticos capaces de expresar ese sentido originario.

\section{Conclusión}

A partir de la publicación del libro de Gondek y Tengelyi parece existir cierto acuerdo respecto de la afirmación del carácter acontecial como el rasgo que define el nuevo estatuto del fenómeno. Y, sin embargo, la definición de fenómeno como "acontecimiento de sentido", propuesta por estos autores, no es aplicable con el mismo grado de adecuación a todos los "nuevos fenomenólogos". Como bien señala Claudia Serban, de las propuestas filosóficas analizadas por Gondek y Tengelyi, es la de Richir la que mejor se adapta a la definición sugerida, pues sostiene la teoría de la formación del "sentido que se hace" (sens sefaisant), que adviene como "acontecimiento". Pero, según Serban, es más problemático hablar de esta dimensión de "sentido", que convoca a

18 Dice Marion "Si una cuestión dotada de sentido, correctamente formulada y sin contradicción lógica, queda sin respuesta posible para un espíritu finito, e incluso no debe, por razones a priori, recibir respuesta según los criterios de una racionalidad finita (metafísica, los dos principios de contradicción y de razón suficiente), entonces esa cuestión en cuanto siempre es buscada y siempre dejada sin respuesta, que sobrevive sin embargo a esta ausencia ¿acaso no da ella una realidad que pensar (cogitable) y no merece el rango de certeza negativa? Pues incluso la denegación puede concernir a la donación" (2010: 19-20). 
una hermenéutica en Marion y en Henry. Es conocido el rechazo de Henry por la hermenéutica, a la que considera una deriva indeseable de la fenomenología (cfr. 1990: 8, y 1996: 282). Por su parte, Marion casi no había referido a ella de modo explícito hasta la publicación de Reprise du donné (Cfr. Serban, 2016: 31-33). En este sentido, la consideración del estatuto que estas propuestas le otorgan a la instancia antepredicativa, entendida en el sentido más amplio de un ámbito prelingüístico, permite develar un rasgo común que las inscribe de modo definitivo en la "escuela fenomenológica". Heidegger define la fenomenología como la "permanente posibilidad del pensar de corresponderse con la exigencia de aquello que hay que pensar" (GA 14: 101). ¿Cómo es posible que se dé esta correspondencia? ¿Cómo acceder a esa exigencia que proviene del fenómeno? Sencillamente, otorgando la iniciativa al fenómeno, aceptando su carácter acontecial. ¿Y esto qué implica? ¿Qué significa concretamente aceptar la donación del fenómeno como un acontecimiento? Significa entregarse a su sentido, prescindir de la operación de Sinngebung para mantenerse receptivo respecto del sentido que el fenómeno se da a sí mismo; ese sentido prelingüístico que se articula en la experiencia cuando "hacemos" una experiencia en lugar de "tenerla". Esto es lo que la nueva fenomenología francesa comprende: volver a las cosas mismas implica dejar que las cosas mismas aparezcan en sus propios términos. Y esto significa ser capaz de recibir (como una exigencia) el sentido antepredicativo o incluso no-predicativo (no traducible a términos predicativos) que se da en la experiencia con el fenómeno.

El análisis de las reflexiones de Romano y de Marion permite advertir los dos rasgos fundamentales del nuevo estatuto de lo antepredicativo en la fenomenología francesa actual: 1) la importancia otorgada a la afirmación de un sentido que no es introducido en la experiencia por un "marco kantiano" que imponga "construcciones desde arriba", como el rasgo fenomenológico más propio (tesis fenomenológica de Romano), y que no siempre es reconducible a la esfera teórica, sino que muestra su validez originaria en el campo de la práctica, y 2) el intento de explorar otros actos lingüísticos que permitan encontrar una lingüisticidad para una experiencia que no se deja reconducir a la objetidad ni al ámbito teórico del lenguaje predicativo (los modelos de la plegaria o la alabanza en Marion y en Levinas).

Ciertamente, habría que continuar la indagación respecto de otros autores, y demostrar la aplicabilidad de esta tesis a sus propuestas, pero cabe especular que los fenómenos de la carne en Henry, la promesa en Chrétien, el acontecimiento en Maldiney, por nombrar solo algunos, despliegan también un sentido preteórico que demanda ser aprehendido en sus propios términos, y se resiste a ser reconducido al plano teórico predicativo. 


\section{Bibliografía}

" Ainbinder, B. y Stanciu, O. (2020). Metabolé des Vorprädikativen. Faktizität und die ontischen Wurzeln der Prädikation. En Pasqualin, C. y Sforza, A. (eds), Das Vorprädikative. Perspektiven im Ausgang von Heidegger. Freiburg: Verlag Karl Alber.

» Bassas Vila, J. (2017). El 'lenguaje saturado' de Jean-Luc Marion: ¿de la fenomenología a la política? En Roggero, J. L. (ed.), Jean-Luc Marion. Límites y posibilidades de la filosofía y de la teología (211-226). Buenos Aires: SB Editorial.

" Bégout, B. (2000). La généalogie de la logique. Husserl, et le catégorial. París: Vrin.

"Bégout, B. (2008). Le phénomène et son ombre. Chatou: La Transparence.

» Benoist, J. (2001 [1998]). Sur l'état présent de la phénoménologie. En Benoist, J., L'idée de phénoménologie. París: Beauchesne.

"Canullo, C. (2004). La fenomenologia rovesciata. Percosi tentati in Jean-Luc Marion, Michel Henry e Jean-Louis Chrétien. Torino: Rosenberg e Sellier.

» Falque, E. (2015 [2004]). Métamorphose de la finitude. En Falque, E., Triduum philosophique. París: Cerf.

» Falque, E. (2014). Le combat amoureux. Disputes phénoménologiques et théologiques. París: Hermann.

» Gondek, H-D y Tengeleyu, L. (2011). Neue Phänomenologie in Frankreich. Berlin: Suhrkamp.

» Heidegger, M. (1979 [1925]). Gesamtausgabe. Il. Abteilung. Vorlesungen 19231944. Band 20. Prolegomena zur Geschichte des Zeitbegriffs. Frankfurt am Main: Vittorio Klostermann (GA 20).

»Heidegger, M. (1983 [1929]). Gesamtausgabe. II. Abteilung. Vorlesungen 19231944. Band 29/30. Die Grundbegriffe der Metaphysik. Welt, Endlichkeit, Einsamkeit, Frankfurt am Main, Vittorio Klostermann (GA 29/30).

»Heidegger, M. (1985 [1950-1959]). Gesamtausgabe. l. Abteilung: Veröffentlichte Schriften 1910-1976. Band 12. Unterwegs zur Sprache, Frankfurt am Main, Vittorio Klostermann (GA 12).

"Heidegger, M. (2007 [1969]). Gesamtausgabe. I. Abteilung: Veröffentlichte Schriften 1910-1976. Band 14. Zur Sache des Denkens. Frankfurt am Main : Vittorio Klostermann (GA 14).

» Henry, M. (1990). Phénoménologie matérielle. París: PUF.

» Henry, M. (1996). C'est moi la vérité: pour une philosophie du christianisme. París : Seuil.

" Husserl, E. (1984 [1901]). Husserliana. Edmund Husserl Gesammelte Werke. Band $X I X / 2$. Logische Untersuchungen. Zweiter Band. Zweiter Teil. Untersuchungen zür Phänomenologie und Theorie der Erkenntnis. Haag: Martinus Nihjoff (Hua XIX/2).

» Husserl, E. (1952 [1912]). Husserliana. Edmund Husserl Gesammelte Werke. Band V. Ideen zu einer reinen Phänomenologie und Phänomenologischen Philosophie. Drittes Buch. Die Phänomenologie und die Fundamente der Wissenschaften, Haag, Martinus Nihjoff (Hua V).

» Husserl, E. (1939 [1913]). Entwurf einer 'Vorrede' zu den 'Logischen Untersuchungen' (1913) (Hrsg. v. E. Fink), Tijdschrift voor Filosofie 1, 106-133. 
" Husserl, E. (1974 [1929]). Husserliana. Edmund Husserl Gesammelte Werke. Band XVII: Formale und transzendentale Logik, Haag, Martinus Nihjoff (Hua XVII).

» Husserl, E. (1976 [1936]). Husserliana. Edmund Husserl Gesammelte Werke. Band VI. Die Krisis der Europäischen Wissenschaften und die Transzendentale Phänomenologie. Ein Einleitung in die Phänomenologische Philosophie. Haag; Martinus Nihjoff (Hua VI).

» Husserl, E. (1964 [1939]). Erfahrung und Urteil. Untersuchungen zur Genealogie der Logik, (redigiert und herausgegeben von Ludwig Landgrebe), Hamburg: Classen Verlag.

" Janicaud, D. (2009 [1991]). Le tournant théologique de la phénoménologie française. En Janicaud, D., La phénoménologie dans tous ses états. París: Gallimard.

» Janicaud, D. (2009 [1998]). La phénoménologie éclatée. En Janicaud, D., La phénoménologie dans tous ses états. París: Gallimard.

» Levinas, E. (1991 [1951]). L'ontologie, est-elle fondamentale? En Levinas, E. Entrenous. Essais sur le penser-à-l'autre. París: Grasset.

» Levinas, E. (1990 [1961]). Totalité et Infini. Essai sur l'extériorité. París: Le livre de poche.

" Lohmar, D. (1996). La genèse du jugement antéprédicatif dans les Recherches Logiques et dans Expérience et Jugement. En Courtine, J-F (ed.), Phénoménologie et logique (217-238). París: Presses ÉNS.

" Marion, J-L. (1989). Réduction et donation. Recherches sur Husserl, Heidegger et la phénoménologie. París: PUF.

" Marion, J-L. (2002). Un moment français de la phénoménologie, Rue Descartes, $35,1,9-13$

" Marion, J-L. (2008). Au lieu de soi. L'approche de Saint Augustin. París: PUF.

" Marion, J-L. (2010). Certitudes négatives. París: Grasset.

" Marion, J-L. (2012). La rigueur des choses. Entretiens avec Dan Arbib. París: Flammarion.

» Pizzi, M. I. (2019). Exploraciones sobre el lenguaje no predicativo en la fenomenología de Jean-Luc Marion, Dionisio Areopagita y Friedrich Nietzsche, Revista Litura, 2, 1-13.

"Pizzi, M. I. (2020). La certeza negativa a la luz del vocablo cusano possest. aportes para un lenguaje de la saturación. En Roggero, J. L. (ed.), El fenómeno saturado. La excedencia de la donación en la fenomenología de Jean-Luc Marion (pp. 191-200). Buenos Aires: SB Editorial.

» Ricœur, P. (1986 [1975]). Phénoménologie et herméneutique. En venant de Husserl. En Ricœur, P., Du texte à l'action. Essais d'herméneutique II (pp. 43-81). París: Éditions du Seuil.

"Roggero, J. L. (2019). Hermenéutica del amor. La fenomenología de la donación de Jean-Luc Marion en diálogo con la fenomenología del joven Heidegger. Buenos Aires: Sb Editorial.

» Romano, C. (1998). L'événement et le monde, París: PUF.

» Romano, C. (2010). Au cœur de la raison, la phénoménologie. París: Gallimard.

» Sebbah, D-F (2001). À l'épreuve de la limite. Derrida, Henry, Lévinas et la phénoménologie. París: PUF. 
"Serban, C. (2016). La "nouvelle phénoménologie" en France et les événements de sens (Sinnereignisse). Un prolongement de la lecture de László Tengelyi. En Cabestan, P. (ed.), L'événement et la raison. Autour de Claude Romano (pp. 31-45). París: Le Cercle Herméneutique.

» Thomas-Fogiel, I. (2015). Le lieu de l'universel. Impasses du réalisme dans la philosophie contemporaine. París: Seuil.

»Vinolo, S. (2019). Jean-Luc Marion, apologie de l'inexistence. Tome II. Une phénoménologie discursive. París: L'Harmattan. 
